Journal of Universal Mathematics

VOL.4 No.1 PP.13-24 (2021)

ISSN-2618-5660

DOI: $10.33773 /$ jum. 796173

\title{
A NEW PERSPECTIVE ON EVALUATION SYSTEM IN EDUCATION WITH INTUITIONISTIC FUZZY LOGIC AND PROMETHEE ALGORITHM
}

\author{
FERIDE TUĞRUL* AND MEHMET ÇİTIL
}

\begin{abstract}
This research attempts to seek for multi criteria decision making through the PROMETHEE method. This paper aims to propose an application of multi criteria decision making in intuitionistic fuzzy sets. The main goal of the paper; to establish a smart evaluation system in education with a multi criteria decision making approach using intuitionistic fuzzy logic and PROMETHEE method. The success ranking of schools was analyzed through the use of the PROMETHEE method. Besides, the most successful school was identified among these ranked schools. In this research, the intuitionistic fuzzy PROMETHEE method was employed during ranking of schools' success. The main reason for using intuitionistic fuzzy PROMETHEE is that it takes into account both intuitionistic fuzzy weights and intuitionistic fuzzy preferences at the same time. This paper applies to many fields; however, we proposed an application in the field of education in this study. The primary purpose of this paper is expected to create an algorithm of intuitionistic fuzzy PROMETHEE and offer an application example that will shed light on many application areas. Using multi-criteria decision-making methods together with intuitionistic fuzzy logic will help us obtain more rational and more objective results.
\end{abstract}

\section{INTRODUCTION}

Fuzzy logic was firstly defined by Zadeh in 1965 [23]. Then, intuitionistic fuzzy sets (shortly IFS), an extension of fuzzy sets, were defined by K.Atanassov in 1986 [3], [4]. IFS theory is advantageous in various application areas. Many applications of intuitionistic fuzzy set have been carried out through distance measures approach. Many researchers have explored applications of the intuitionistic fuzzy set such as medical diagnosis, medical application, career determination, real life situations, education, artificial intelligence, networking. Decision making is the action of selecting between two or more options. Multi criteria decision making (MCDM) is a well known notion that aims to choose the best solution among various alternatives in decision making. The working style of all MCDM methods

Date: September, 2020.

2000 Mathematics Subject Classification. 03E72,90B50.

Key words and phrases. Evaluation system in education, fuzzy logic, multi criteria decision making, intuitionistic fuzzy sets, PROMETHEE method.

Feride Tuğrul has been supported by the Scientific and Technological Research Council of Turkey (2211/A TUBITAK Domestic PhD Scholarship Program). 
is as follows: the Selection of Criteria, the Selection of Alternatives, the Selection of Aggregation Methods and ultimately the Selection of Alternatives which are based on weights or outranking [16]. Some of the MCDM methods are Analytical Hierarchy Process (AHP), Fuzzy Multi Criteria Decision Making Process, ELECTRE Method, Preference Ranking Organization Method for Enrichment of Evaluations (PROMETHEE), The TOPSIS Method. Bellman and Zadeh were the first researchers who introduced decision making in fuzzy logic. Multi criteria fuzzy decision making has been one of the rapidly growing area in recent years thanks to its practicality. In MCDM problems, usually the best alternative is chosen from those depending on the criteria. Recently, intuitionistic fuzzy logic and decision making have attracted the attention of many authors. In addition, applications of MCDM problem have increased in the intuitionistic fuzzy set [10] and we have studies on the applications of intuitionistic fuzzy logic and we continue to improve our work $[25],[26],[27],[28],[29]$.

PROMETHEE method was firstly defined in 1982 [5]. PROMETHEE method provides taken partial or complete ranking of alternatives based on the positive outranking flow, the negative outranking flow as well as the net outranking flow [15]. After being defined in the fuzzy field, the method has been expanded in the sense of intuitionistic fuzzy. It is much more appropriate to use intuitionistic fuzzy since it includes membership, non-membership and degree of hesitancy. One of the multi-criteria decision-making methods, the PROMETHEE was developed by Jean Pierre Brans (1982), which was then developed by adding PROMETHEE-I and PROMETHEE-II methods [5]. This method differs from other decision methods as it evaluates each alternative within itself. Many researchers have developed applications by means of PROMETHEE methods [1], [2], [7], [8], [9], [11], [12], [24]. It is of highly significant to use the IF PROMETHEE in case of uncertainty or hesitation. Thus, this paper aims to determine the ranking of school success and choose the most successful school depending some criteria. This paper deployed the IF PROMETHEE method since it produces a partial or complete ranking of alternatives both the positive outranking flow and negative outranking flow. IF PROMETHEE gives not only IF preferences, but also IF weights into account. It is suitable to use the IF PROMETHEE method. Through the use of the IF PROMETHEE method, the success of the schools was ranked by taking into account the students' achievements and the most successful school was chosen in the present paper. This paper takes into account the schools located in Kahramanmaraş in Turkey. One assessment point was determined by the researchers for each school depending on the students' examination scores. This research utilized official data that were obtained from the Ministry of Education. We used intuitionistic fuzzy sets as a tool since it incorporates the marks of the questions that have been correctly answered by the students, those that have been wrongly answered and the ones that are free from any answer. There are five alternatives representing schools which were randomly selected from different regions in Kahramanmaraş. This study can be extended to a larger area and can be applied on the basis of schools in all provinces. This paper aims to be an exemplary application for further studies. Official examinations are regularly organized by the Ministry of Education every year. The average score of the students in the official examination was calculated and an assessment point was calculated for each school. The official examination consists of six basic lessons. Thus, the criteria were determined as the 
basic lessons such as Turkish, Mathematics, Science, Social, English, Religion. In brief; education in all countries and especially in our country is a serious issue that needs to be emphasized. In line with the evolving conditions, opportunities and technologies, both the education system, the stages of assessment, selection and decision making, and the reorganization and restructuring of the physical space are deemed necessary. In this study, we used a method that we think will create a model for selection and decision making. Also Covid-19 process; It showed us that it is important to anticipate, to be able to make fast and accurate decisions, and to prepare the infrastructure. Therefore, such a study showed its importance in terms of maintaining the education process well and properly. At the same time, in this process, the lack of infrastructure, making the right decisions against many criteria and alternatives, emphasized the importance of such studies in terms of both material and evaluation. In addition, fuzzy logic and intuitionistic fuzzy logic have been understood to give good results in multi-criteria decision making.

\section{PRELIMINARIES}

Definition 2.1. $([4,3])$ Let $X \neq \emptyset$. An intuitionistic fuzzy set $A$ in $X$;

$$
\begin{aligned}
& A=\left\{\left\langle x, \mu_{A}(x), \nu_{A}(x)\right\rangle \mid x \in X\right\}, \\
& \mu_{A}(x), \nu_{A}(x), \pi_{A}(x): X \rightarrow[0,1]
\end{aligned}
$$

defined membership degree, nonmembership degree and hesitation degree of the element $x \in X$ respectively.

$$
\mu_{A}(x)+\nu_{A}(x)+\pi_{A}(x)=1 .
$$

IF statement will be used instead of intuitionistic fuzzy for brevity throughout this study. Intuitionistic fuzzy value (IFV) defined by Xu ([20]). Intuitionistic fuzzy value $(\mathrm{IFV})$ is shown as follows: $\tilde{a}=\left(\mu_{\tilde{a}}, \nu_{\tilde{a}}, \pi_{\tilde{a}}\right)$, where $\mu_{\tilde{a}}, \nu_{\tilde{a}}, \pi_{\tilde{a}} \in[0,1]$

For each IFS $\tilde{A}$;

$$
\pi_{\tilde{A}}=1-\mu_{\tilde{A}}-\nu_{\tilde{A}}
$$

In this paper; we will not write the third part so we will show $\tilde{a}=\left(\mu_{\tilde{a}}, \nu_{\tilde{a}}\right)$ shape instead of $\tilde{a}=\left(\mu_{\tilde{a}}, \nu_{\tilde{a}}, \pi_{\tilde{a}}\right)$. The degree of hesitation can be obtained by equation 2.1 .

Some equations have been given for IFVs $\tilde{a}=\left(\mu_{\tilde{a}}, \nu_{\tilde{a}}\right)$ and $\tilde{b}=\left(\mu_{\tilde{b}}, \nu_{\tilde{b}}\right)([20,19])$ :

Many researchers have proposed some formulas in order to compare the IFVs $([17,19])$. We will use the following method in this paper. This method has been proposed by Szmidt and Kacprzyk, which gives more logical results compared to other methods $([17])$. This function is used to rank IFVs:

$$
\rho(\alpha)=\frac{\left(\left(1+\pi_{\alpha}\right)\left(1-\mu_{\alpha}\right)\right)}{2}
$$

As the $\rho(\alpha)$ value decreases, the preferred value $\alpha$ increases.

\section{IF PROMETHEE}

When a MCDM is encountered, the decision maker is expected to choose the best alternative among the alternatives according to certain criteria. But the importance of all criteria may vary. In these cases, the weights of criteria are taken into account. The benefit of the PROMETHEE method is to assess considering the weight of the criterion. The criteria's weights indicate how important they are. 
Considering both intuitionistic fuzzy sets and weights of criteria at the same time, more consistent and rational results will be obtained. Therefore; using intuiitonistic fuzzy PROMETHEE method will provide advantageous results. The criteria's weights could be depicted as IFVs: $\tilde{w}_{j}$ where $\mu_{\tilde{w}_{j}} \in[0,1], \nu_{\tilde{w}_{j}} \in[0,1], \mu_{\tilde{w}_{j}}+\nu_{\tilde{w}_{j}} \leq$ $1, j=1,2, \ldots, m$. According to the weights, $\mu_{\tilde{\omega}_{j}}$ and $\nu_{\tilde{\omega}_{j}}$ demonstrate the membership and non-membership degrees of the alternative $x_{i}$ respectively. Indeed; the concept of weight represents the importance of that criteria. The weights are expressed as IFV in the intuitionistic fuzzy PROMETHEE. Some methods can help decision makers in determining intuitionistic fuzzy weights ([?, ?, 18, 22]). In this research, V shape criterion type has been used ([6]):

$$
P(d)= \begin{cases}0, & d \leq q \\ \frac{d-q}{p-q}, & q<d \leq p \\ 1, & d>p\end{cases}
$$

Parameter thresholds $q$ and $p$ are indicated as indifference and strict preference, respectively. Decision makers are free to change these thresholds according to the desired situation. Evaluate the alternatives $x_{i}(i=1,2, \ldots, n)$ with respect to the criteria $c_{j}(j=1,2, \ldots, m)$ and determine the deviations based on pairwise comparisons:

$$
d_{j}(x, y)=c_{j}(x)-c_{j}(y)
$$

where $d_{j}(x, y)$ shows the distinction between the alternatives' the assessments $x$ and $y$ on the criterion $c_{j}$.

3.1. The Relationship Between Intuitionistic Fuzzy and PROMETHEE. In PROMETHEE, the preferences are limited in $[0,1]$ by the generalized criterion. If these preference functions are handled with a fuzzy set, membership functions and preference values are represented by $P_{j}(x, y)$. Besides preference values can be taken directly as fuzzy numbers as they are limited to $[0,1]$. However, with the help of the fuzzy set, only the preferred density defined by the membership function can be expressed. It is more convenient for the decision maker to use intuitionistic fuzzy set because it addresses all aspects of the criteria. In addition, the intuitionistic fuzzy set is advantageous for the PROMETHEE method, since it includes the preferred, undesirable and uncertainty at the same time.

Definition 3.1. ([21]) An IF preference relation $R$ on the set $X$ is symbolized by a matrix $R=\left(r_{i k}\right)_{n \times n}$, where $r_{i k}=\left\langle\left(x_{i}, x_{k}\right), \mu\left(x_{i}, x_{k}\right), \nu\left(x_{i}, x_{k}\right)\right\rangle$ for all $i, k=$ $1,2, \ldots, n$. For convenience, we let $r_{i k}=\left(\mu_{i k}, \nu_{i k}\right)$ where $\mu_{i k}$ denotes the degree to which the object $x_{i}$ is preferred to the object $x_{k}, \nu_{i k}$ indicates not preferred and $\pi\left(x_{i}\right.$ indicates as a degree of hesitancy, provided that:

$$
\begin{gathered}
\mu_{i k}, \nu_{i k} \in[0,1], \mu_{i k}+\nu_{i k} \leq 1, \mu_{i k}=\nu_{k i}, \mu_{k i}=\nu_{i k} \\
\mu_{i i}=\nu_{i i}=0.5, \pi_{i k}=1-\mu_{i k}-\nu_{i k} \\
\text { for all } i, k=1,2, \ldots, n
\end{gathered}
$$




\section{Algorithm of Intuitionistic Fuzzy PROMETHEE}

The preferences $\mu_{i k}$ between the alternatives $x_{i}$ and $x_{k}$ according to the criterion $c_{j}$ could be determined by (3.2) and (3.1), and then the preference matrix according to the criterion $c_{j}$ is determined as ([?]):

$$
U^{(j)}=\left(\mu_{i k}^{(j)}\right)_{n \times n}=\left[\begin{array}{cccc}
- & \mu_{12}^{(j)} & \ldots & \mu_{1 n}^{(j)} \\
\mu_{21}^{(j)} & - & \ldots & \mu_{2 n}^{(j)} \\
\vdots & \vdots & - & \vdots \\
\mu_{n 1}^{(j)} & \mu_{n 2}^{(j)} & \ldots & -
\end{array}\right]
$$

Using the equations $\nu_{k i}=\mu_{i k}$ and $\nu_{i k}=\mu_{k i}$, matrix of the IF preference relation is obtained:

$$
R^{(j)}=\left(r_{i k}^{(j)}\right)_{n \times n}=\left[\begin{array}{cccc}
- & \left(\mu_{12}^{(j)}, \nu_{12}^{(j)}\right) & \ldots & \left(\mu_{1 n}^{(j)}, \nu_{1 n}^{(j)}\right) \\
\left(\mu_{21}^{(j)}, \nu_{21}^{(j)}\right) & - & \ldots & \left(\mu_{2 n}^{(j)}, \nu_{2 n}^{(j)}\right) \\
\vdots & \vdots & - & \vdots \\
\left(\mu_{n 1}^{(j)}, \nu_{n 1}^{(j)}\right) & \left(\mu_{n 2}^{(j)}, \nu_{n 2}^{(j)}\right) & \ldots & -
\end{array}\right]
$$

Then; considering the $c_{j}(j=1,2, \ldots, m)$ criteria, we must establish the general preference index for each alternative. We can get what we want by using weighted aggregation operators. We will use the IFWA operator in this paper $([20,19])$. The IF preference index of the alternative $x_{i}$ to $x_{k}$ on criteria could be obtained as:

$$
r\left(x_{i}, x_{k}\right)=r_{i k}=\bigoplus_{j=1}^{m}\left(\tilde{w}_{j} \bigotimes r_{i k}^{(j)}\right)
$$

where $r\left(x_{i}, x_{k}\right)=r_{i k}$ shows the degree to which the alternative $x_{i}$ is preferred to the alternative $x_{k}$ all criteria. Also, $r_{i k}$ is an IFV. $\tilde{w}_{j}=\left(\mu_{\tilde{w} j}, \nu_{\tilde{w} j}\right)$ :

$$
\tilde{w}_{j} \bigotimes r_{i k}^{(j)}=\left(\mu_{i k}^{(j)} \mu_{\tilde{w} j}, \nu_{i k}^{(j)}+\nu_{\tilde{w} j}-\nu_{i k}^{(j)} \nu_{\tilde{w} j}\right)
$$

If Equations ((4.3) and (4.4) are combined;

$$
\begin{aligned}
r\left(x_{i}, x_{k}\right)= & \bigoplus_{j=1}^{m}\left(\tilde{w}_{j} \bigotimes r_{i k}^{(j)}\right) \\
= & \left(1-\prod_{j=1}^{m}\left(1-\mu_{i k}^{(j)} \mu_{\tilde{w} j}\right),\right. \\
& \left.\prod_{j=1}^{m}\left(\nu_{i k}^{(j)}+\nu_{\tilde{w} j}-\nu_{i k}^{(j)} \nu_{\tilde{w} j}\right)\right)
\end{aligned}
$$

Overall IF preference relationship is established as follows:

$$
R=\left(r_{i k}\right)_{n \times n}=\left[\begin{array}{cccc}
- & \left(\mu_{12}, \nu_{12}\right) & \ldots & \left(\mu_{1 n}, \nu_{1 n}\right) \\
\left(\mu_{21}, \nu_{21}\right) & - & \ldots & \left(\mu_{2 n}, \nu_{2 n}\right) \\
\vdots & \vdots & - & \vdots \\
\left(\mu_{n 1}, \nu_{n 1}\right) & \left(\mu_{n 2}, \nu_{n 2}\right) & \ldots & -
\end{array}\right]
$$

The number of alternatives against which each alternative is compared is $(n-1)$. As a result of IF positive outranking flow and IF negative outranking flow can be 
achieved as follows:

(1) The IF positive outranking flow:

$$
\tilde{\varphi}^{+}\left(x_{i}\right)=\frac{1}{n-1} \bigoplus_{k=1, k \neq i}^{n} r_{i k}
$$

(2) The IF negative outranking flow:

$$
\tilde{\varphi}^{-}\left(x_{i}\right)=\frac{1}{n-1} \bigoplus_{k=1, k \neq i}^{n} r_{k i}
$$

The relationship between $\tilde{\varphi}^{+}\left(x_{i}\right)$ and $\tilde{\varphi}^{-}\left(x_{i}\right)$ can be explained with the help of Equation (2.2). The IF net cannot be obtained by directly subtraction the outranking flow. The distinction between the IF positive outranking flow and the IF negative outranking flow could be calculated using the function defined by Szmidt and Kacprzyk.

$$
\rho\left(\varphi\left(x_{i}\right)\right)=\rho\left(\tilde{\varphi}^{+}\left(x_{i}\right)\right)-\rho\left(\tilde{\varphi}^{-}\left(x_{i}\right)\right)
$$

Three different ranking for $\tilde{\varphi}^{+}\left(x_{i}\right)$ and $\tilde{\varphi}^{-}\left(x_{i}\right)$ can be achieved ([?]):

(1) Partial ranking: $x_{i}$ outranks $x_{k}$ if $\tilde{\varphi}^{+}\left(x_{i}\right) \geq \tilde{\varphi}^{+}\left(x_{k}\right)$ and $\tilde{\varphi}^{-}\left(x_{i}\right) \leq \tilde{\varphi}^{-}\left(x_{k}\right)$;

(2) Equality: $\tilde{\varphi}^{+}\left(x_{i}\right)=\tilde{\varphi}^{+}\left(x_{k}\right)$ and $\tilde{\varphi}^{-}\left(x_{i}\right)=\tilde{\varphi}^{-}\left(x_{k}\right)$ indicates indifference between alternatives.

(3) Incomparability: If $\tilde{\varphi}^{+}\left(x_{i}\right)>\tilde{\varphi}^{+}\left(x_{k}\right)$ and $\tilde{\varphi}^{-}\left(x_{i}\right)>\tilde{\varphi}^{-}\left(x_{k}\right)$ or $\tilde{\varphi}^{+}\left(x_{i}\right)<$ $\tilde{\varphi}^{+}\left(x_{k}\right), \tilde{\varphi}^{-}\left(x_{i}\right)<\tilde{\varphi}^{+}\left(x_{k}\right)$.

If incomparability happens, IF net outranking flow could be obtained by the help of Equation (4.9).

The algorithm for the intuitionistic fuzzy PROMETHEE could be set up as ([?]): Here the algorithm will provide convenience during application.

Step 1: Determine alternatives $X=x_{1}, x_{2}, \ldots, x_{n}$ and criteria $C=c_{1}, c_{2}, \ldots, c_{m}$. Step 2: State by degrees how important the criteria are $\tilde{w}_{j}(j=1,2, \ldots, m)$ where $\mu_{\tilde{w}_{j}}+\nu_{\tilde{w}_{j}} \leq 1, \mu_{\tilde{w}_{j}} \in[0,1], \nu_{\tilde{w}_{j}} \in[0,1]$

Step 3: Determine $q$ and $p$ parameters as an indifference threshold and a strict preference threshold, respectively. Calculate deviations $d_{j}(x, y)$ using Equation (3.2). Calculate the preferences $\mu_{i k}^{(j)}$. Then, $U^{(j)}(j=1,2, \ldots, m)$ the preference matrix could be created.

Step 4: Create the IF preference relation $R^{(j)}=\left(r_{i k}^{(j)}\right)_{n \times n}$.

Step 5: Create the overall IF preference relation $R=\left(r_{i k}\right)_{n \times n}$ using Equation (4.3).

Step 6: Determine the IF positive outranking flow $\tilde{\varphi}^{+}\left(x_{i}\right)$ and the IF negative outranking flow $\tilde{\varphi}^{-}\left(x_{i}\right)$ by using Equation (4.5) and (4.6).

Step 7: The relationship between $\tilde{\varphi}^{+}\left(x_{i}\right)$ and $\tilde{\varphi}^{-}\left(x_{i}\right)$ is determined. According to this relationship is made a ranking.

Step 8: End.

\section{Application of Intuitionistic Fuzzy PROMETHEE}

Step 1: This paper aims to determine the ranking of school success and choose the most successful school depending some criteria. Through use of intuitionistic fuzzy PROMETHEE method, the success of the schools was ranked by taking into

account the students' achievements and the most successful school was chosen in 
the present paper. We used IFS as a tool since it incorporates the marks of the questions that have been correctly answered by the students those that have been wrongly answered and the ones that are free from any answer. There are five alternatives representing schools which were randomly selected from different regions in Kahramanmaraş. The average score of the students in the official examination was calculated and an assessment point was calculated for each school. The official examination consists of six basic lessons. Thus, the criteria were determined as the basic lessons such as Turkish, Mathematics, Science, Social, English, Religion.

$H=\left\{H_{1}, H_{2}, H_{3}, H_{4}, H_{5}\right\}$ be set of schools.

$L=\left\{L_{1}, L_{2}, L_{3}, L_{4}, L_{5}, L_{6}\right\}$ be set of criteria. Set of criteria respectively are $L=\{$ Turkish, Mathematics, Science, Social,English, Religion $\}$.

\begin{tabular}{|c|l|l|l|l|l|l|}
\hline Alternatives & $L_{1}$ & $L_{2}$ & $L_{3}$ & $L_{4}$ & $L_{5}$ & $L_{6}$ \\
\hline$H_{1}$ & 19.2 & 19 & 19.9 & 19.4 & 19 & 19.9 \\
\hline$H_{2}$ & 18.4 & 18.6 & 18.6 & 18 & 18.2 & 19.6 \\
\hline$H_{3}$ & 17 & 16.4 & 15 & 17 & 16.2 & 18 \\
\hline$H_{4}$ & 13 & 11 & 13 & 16.2 & 12.6 & 11 \\
\hline$H_{5}$ & 10 & 6 & 12.6 & 13.2 & 13 & 16.2 \\
\hline
\end{tabular}

Step 2: In the official examinations, the contribution of each lesson to the exam result score is equal. In other words, the importance of criteria is of equal weight. Weights of criteria in IFVs; $\tilde{w}_{j}(j=1,2, \ldots, 6)$

\begin{tabular}{|l|l|}
\hline$\tilde{w}_{1}=(0.167,0.1)$ & $\tilde{w}_{4}=(0.167,0.1)$ \\
\hline$\tilde{w}_{2}=(0.167,0.1)$ & $\tilde{w}_{5}=(0.167,0.1)$ \\
\hline$\tilde{w}_{3}=(0.167,0.1)$ & $\tilde{w}_{6}=(0.167,0.1)$ \\
\hline
\end{tabular}

where $\mu_{\tilde{w}_{j}}, \nu_{\tilde{w}_{j}} \in[0,1],(j=1,2, \ldots, 6)$.

Step 3: Deviation $d_{j}\left(x_{i}, x_{k}\right)$ for each criterion; can be calculated using V-shape generalization criterion. For V-shape generalization criterion, the indifference threshold $q$ is determined equal to 20 and $p$ strict preference threshold is calculated equal to 0 for all criteria. This is the threshold determined by the researcher or the decision maker. Threshold calculations may vary for each research.

$$
\begin{gathered}
d_{j}\left(x_{i}, x_{k}\right)=c_{j}\left(x_{i}\right)-c_{j}\left(x_{k}\right) \\
\mu_{i k}^{(j)}= \begin{cases}0, & d_{j}\left(x_{i}, x_{k}\right) \leq q \\
\frac{d_{j}\left(x_{i}, x_{k}\right)-q}{p-q}, & q<d_{j}\left(x_{i}, x_{k}\right) \leq p \\
1, & d_{j}\left(x_{i}, x_{k}\right)>p\end{cases}
\end{gathered}
$$

where $p=20, q=0$ are given. The preference matrices $U^{(j)}(j=1,2,3,4,5,6)$ are determined as follows:

$$
U^{(1)}=\left[\begin{array}{ccccc}
- & 0.04 & 0.11 & 0.31 & 0.46 \\
0 & - & 0.07 & 0.27 & 0.42 \\
0 & 0 & - & 0.2 & 0.35 \\
0 & 0 & 0 & - & 0.15 \\
0 & 0 & 0 & 0 & -
\end{array}\right]
$$




$$
\begin{aligned}
U^{(2)} & =\left[\begin{array}{ccccc}
- & 0.08 & 0.17 & 0.4 & 0.65 \\
0 & - & 0.11 & 0.38 & 0.63 \\
0 & 0 & - & 0.27 & 0.52 \\
0 & 0 & 0 & - & 0.25 \\
0 & 0 & 0 & 0 & -
\end{array}\right] \\
U^{(3)} & =\left[\begin{array}{ccccc}
- & 0.065 & 0.245 & 0.345 & 0.365 \\
0 & - & 0.18 & 0.28 & 0.3 \\
0 & 0 & - & 0.1 & 0.12 \\
0 & 0 & 0 & - & 0.02 \\
0 & 0 & 0 & 0 & -
\end{array}\right] \\
U^{(4)} & =\left[\begin{array}{ccccc}
- & 0.07 & 0.12 & 0.16 & 0.31 \\
0 & - & 0.05 & 0.09 & 0.24 \\
0 & 0 & - & 0.04 & 0.19 \\
0 & 0 & 0 & - & 0.15 \\
0 & 0 & 0 & 0 & -
\end{array}\right] \\
U^{(5)} & =\left[\begin{array}{ccccc}
- & 0.04 & 0.14 & 0.32 & 0.3 \\
0 & - & 0.2 & 0.28 & 0.26 \\
0 & 0 & - & 0.18 & 0.16 \\
0 & 0 & 0 & - & 0 \\
0 & 0 & 0 & 0.02 & -
\end{array}\right] \\
U^{(6)} & =\left[\begin{array}{ccccc}
- & 0.015 & 0.095 & 0.445 & 0.185 \\
0 & - & 0.08 & 0.43 & 0.17 \\
0 & 0 & - & 0.35 & 0.09 \\
0 & 0 & 0 & - & 0 \\
0 & 0 & 0 & 0.26 & -
\end{array}\right]
\end{aligned}
$$

Step 4: The IF preference matrices $R^{(j)}=\left(r_{i k}^{(j)}\right)_{n \times n}$ are determined by benefitted from equation $\nu_{i k}=\mu_{k i}$ and $\nu_{k i}=\mu_{i k}$ :

$$
\begin{array}{r}
R^{(1)}=\left[\begin{array}{ccccc}
- & (0.04,0) & (0.11,0) & (0.31,0) & (0.46,0) \\
(0,0.04) & - & (0.07,0) & (0.27,0) & (0.42,0) \\
(0,0.11) & (0,0.07) & - & (0.2,0) & (0.35,0) \\
(0,0.31) & (0,0.27) & (0,0.2) & - & (0.15,0) \\
(0,0.46) & (0,0.42) & (0,0.35) & (0,0.15) & -
\end{array}\right] \\
R^{(2)}=\left[\begin{array}{ccccc}
- & (0.08,0) & (0.17,0) & (0.4,0) & (0.65,0) \\
(0,0.08) & - & (0.11,0) & (0.38,0) & (0.63,0) \\
(0,0.17) & (0,0.11) & - & (0.27,0) & (0.52,0) \\
(0,0.4) & (0,0.38) & (0,0.27) & - & (0.25,0) \\
(0,0.65) & (0,0.63) & (0,0.52) & (0,0.25) & -
\end{array}\right] \\
R^{(3)}=\left[\begin{array}{ccccc}
- & (0.065,0) & (0.245,0) & (0.345,0) & (0.365,0) \\
(0,0.065) & - & (0.18,0) & (0.28,0) & (0.3,0) \\
(0,0.245) & (0,0.18) & - & (0.1,0) & (0.12,0) \\
(0,0.345) & (0,0.28) & (0,0.1) & - & (0.02,0) \\
(0,0.365) & (0,0.3) & (0,0.12) & (0,0.02) & -
\end{array}\right]
\end{array}
$$




$$
\begin{array}{r}
R^{(4)}=\left[\begin{array}{ccccc}
- & (0.07,0) & (0.12,0) & (0.16,0) & (0.31,0) \\
(0,0.07) & - & (0.05,0) & (0.09,0) & (0.24,0) \\
(0,0.12) & (0,0.05) & - & (0.04,0) & (0.19,0) \\
(0,0.16) & (0,0.09) & (0,0.04) & - & (0.15,0) \\
(0,0.31) & (0,0.24) & (0,0.19) & (0,0.15) & -
\end{array}\right] \\
R^{(5)}=\left[\begin{array}{ccccc}
- & (0.04,0) & (0.14,0) & (0.32,0) & (0.3,0) \\
(0,0.04) & - & (0.2,0) & (0.28,0) & (0.26,0) \\
(0,0.14) & (0,0.2) & - & (0.18,0) & (0.16,0) \\
(0,0.32) & (0,0.28) & (0,0.18) & - & (0,0.02) \\
(0,0.3) & (0,0.26) & (0,0.16) & (0.02,0) & -
\end{array}\right] \\
R^{(6)}=\left[\begin{array}{ccccc}
- & (0.015,0) & (0.095,0) & (0.445,0) & (0.185,0) \\
(0,0.015) & - & (0.08,0) & (0.43,0) & (0.17,0) \\
(0,0.095) & (0,0.08) & - & (0.35,0) & (0.09,0) \\
(0,0.445) & (0,0.43) & (0,0.35) & - & (0,0.26) \\
(0,0.185) & (0,0.17) & (0,0.09) & (0.26,0) & -
\end{array}\right]
\end{array}
$$

Step 5: Overall IF preference relation $R=\left(r_{i k}\right)_{n \times n}$ is created using Equation (4.5);

$$
R=\left[\begin{array}{ccccc}
- & \left(0.0508,1.10^{-6}\right) & \left(0.1384,1.10^{-6}\right) & \left(0.288,1.10^{-6}\right) & \left(0.3254,1.10^{-6}\right) \\
\left(0,9.10^{-6}\right) & - & \left(0.11,1.10^{-6}\right) & \left(0.257,1.10^{-6}\right) & \left(0.294,1.10^{-6}\right) \\
\left(0,1.10^{-4}\right) & \left(0,5.10^{-5}\right) & - & \left(0.177,1.10^{-6}\right) & \left(0.217,1.10^{-6}\right) \\
\left(0,3.10^{-3}\right) & \left(0,1.10^{-3}\right) & \left(0,2.10^{-4}\right) & - & \left(0.092,3.10^{-6}\right) \\
\left(0,5.10^{-3}\right) & \left(0,3.10^{-3}\right) & \left(0,5.10^{-4}\right) & \left(0.046,2.10^{-5}\right) & -
\end{array}\right]
$$

Step 6: The IF positive outranking flows and IF negative outranking flows are calculated using Equation (4.7),(4.8):

\begin{tabular}{|l|l|}
\hline$\tilde{\varphi}^{+}\left(x_{1}\right)=\left(0.1517,0.25 .10^{-26}\right)$ & $\tilde{\varphi}^{-}\left(x_{1}\right)=\left(0,0.337 .10^{-16}\right)$ \\
\hline$\tilde{\varphi}^{+}\left(x_{2}\right)=\left(0.1332,0.225 .10^{-26}\right)$ & $\tilde{\varphi}^{-}\left(x_{2}\right)=\left(0.0127,0.375 .10^{-18}\right)$ \\
\hline$\tilde{\varphi}^{+}\left(x_{3}\right)=\left(0.0888,0.125 .10^{-22}\right)$ & $\tilde{\varphi}^{-}\left(x_{3}\right)=\left(0.0582,0.25 .10^{-21}\right)$ \\
\hline$\tilde{\varphi}^{+}\left(x_{4}\right)=\left(0.023,0.45 .10^{-17}\right)$ & $\tilde{\varphi}^{-}\left(x_{4}\right)=\left(0.1461,0.5 .10^{-25}\right)$ \\
\hline$\tilde{\varphi}^{+}\left(x_{5}\right)=\left(0.0115,0.375 .10^{-15}\right)$ & $\tilde{\varphi}^{-}\left(x_{5}\right)=\left(0.1653,0.75 .10^{-26}\right)$ \\
\hline
\end{tabular}

Above values have ranked using Equation (2.2);

\begin{tabular}{|l|l|}
\hline$\rho\left(\tilde{\varphi}^{+}\left(x_{1}\right)\right)=0.7839$ & $\rho\left(\tilde{\varphi}^{-}\left(x_{1}\right)\right)=0.9995$ \\
\hline$\rho\left(\tilde{\varphi}^{+}\left(x_{2}\right)\right)=0.809$ & $\rho\left(\tilde{\varphi}^{-}\left(x_{2}\right)\right)=0.9749$ \\
\hline$\rho\left(\tilde{\varphi}^{+}\left(x_{3}\right)\right)=0.8707$ & $\rho\left(\tilde{\varphi}^{-}\left(x_{3}\right)\right)=0.9143$ \\
\hline$\rho\left(\tilde{\varphi}^{+}\left(x_{4}\right)\right)=0.9657$ & $\rho\left(\tilde{\varphi}^{-}\left(x_{4}\right)\right)=0.7915$ \\
\hline$\rho\left(\tilde{\varphi}^{+}\left(x_{5}\right)\right)=0.9828$ & $\rho\left(\tilde{\varphi}^{-}\left(x_{5}\right)\right)=0.7657$ \\
\hline
\end{tabular}

The following ranking is obtained;

$$
\begin{aligned}
& \rho\left(\tilde{\varphi}^{+}\left(x_{1}\right)\right)<\rho\left(\tilde{\varphi}^{+}\left(x_{2}\right)\right)<\rho\left(\tilde{\varphi}^{+}\left(x_{3}\right)\right)<\rho\left(\tilde{\varphi}^{+}\left(x_{4}\right)\right)<\rho\left(\tilde{\varphi}^{+}\left(x_{5}\right)\right) \\
& \rho\left(\tilde{\varphi}^{-}\left(x_{1}\right)\right)>\rho\left(\tilde{\varphi}^{-}\left(x_{2}\right)\right)>\rho\left(\tilde{\varphi}^{-}\left(x_{3}\right)\right)>\rho\left(\tilde{\varphi}^{-}\left(x_{4}\right)\right)>\rho\left(\tilde{\varphi}^{-}\left(x_{5}\right)\right)
\end{aligned}
$$

Step 7: According to this;

$$
\tilde{\varphi}^{+}\left(x_{1}\right)>\tilde{\varphi}^{+}\left(x_{2}\right)>\tilde{\varphi}^{+}\left(x_{3}\right)>\tilde{\varphi}^{+}\left(x_{4}\right)>\tilde{\varphi}^{+}\left(x_{5}\right)
$$

and at the same time

$$
\tilde{\varphi}^{-}\left(x_{1}\right)<\tilde{\varphi}^{-}\left(x_{2}\right)<\tilde{\varphi}^{-}\left(x_{3}\right)<\tilde{\varphi}^{-}\left(x_{4}\right)<\tilde{\varphi}^{-}\left(x_{5}\right)
$$


When all alternatives are ranked, the partial ranking is obtained. At the same time, the same result is obtained when the Equation 2.2 is provided.

Step 8: The ranking between alternatives is as follows: $H_{1}>H_{2}>H_{3}>H_{4}>H_{5}$. Accordingly, the best option, that is, the most successful school is $H_{1}$. Thanks to the IF PROMETHEE, both positive outranking flow and negative outranking flow were simultaneously compared.

\section{Conclusion and Suggestions}

This research attempts to seek for MCDM PROMETHEE method. This paper aims to propose an application of MCDM in IFS. The success ranking of schools was analyzed through the use of the PROMETHEE method. Besides, the most successful school was identified among these ranked schools. In this research, intuitionistic fuzzy PROMETHEE method was employed during ranking of schools' success. The main reason for using intuitionistic fuzzy PROMETHEE is that it takes into account both intuitionistic fuzzy weights and intuitionistic fuzzy preferences at the same time. Thus, this paper aims to determine the ranking of school success and choose the most successful school depending some criteria.

The criteria in this paper have been determined as the basic lessons. If desired, other factors (environmental, economic, psychological, etc.) can be added to the criteria. This application can be applied in cases when there is a need to make a choice among alternatives with multi criteria. New research may be carried out by following the steps in the presented algorithm. This paper; it is the first application of the method in education and is a representative template and application. Criteria and weights can be arranged according to the application area. This study can be extended to a larger area and can be applied on the basis of all provinces or other countries. This study is aimed to be an exemplary application for further studies. This study will lead to innovations not only in the field of education but also in many field applications. In this study, data were collected from Turkey, but this study can applicable to any field of education in anywhere because education is universal. This paper is expected to create an algorithm of intuitionistic fuzzy PROMETHEE and offer an application example that will shed light on many application areas. Also Covid-19 process; It showed us that it is important to anticipate, to be able to make fast and accurate decisions, and to prepare the infrastructure. Therefore, such a study showed its importance in terms of maintaining the education process well and properly. At the same time, in this process, the lack of infrastructure, making the right decisions against many criteria and alternatives, emphasized the importance of such studies in terms of both material and evaluation.

\section{REFERENCES}

[1] A. Albadvi, Formulating national information technology strategies: A preference ranking model using PROMETHEE method, European Journal of Operational Research , 153, 290-296, (2004).

[2] A. Albadvi, S.K. Chaharsooghi, A. Esfahanipour, Decision making in stock trading: An application of promethee. European journal of operational research 177, 673-683, (2007).

[3] K.T. Atanassov, More on intuitionistic fuzzy sets. Fuzzy sets and systems 33, 37-45, (1989).

[4] K.T. Atanassov, Intuitionistic fuzzy sets, Springer, pp. 1-137, (1999). 
[5] J.P. Brans, L'ing'eni'erie de la decision; Elaboration d'instruments d'aide a lad'ecision. Lam'ethode PROMETHEE, in: R. Nadeau, M. Landry, ed., L'aide a la d'ecision: Nature, Instruments et Perspectives d'Avenir, Qu'ebec, Canada. Presses de l'Universit'e Laval, 183-213, (1982).

[6] P. Vincke, J.P. Brans, A preference ranking organization method, The PROMETHEE method for Multiple Criteria Decision Making, Management Science 31, 647-656, (1985).

[7] M. Behzadian, R.B. Kazemzadeh, A. Albadvi, M. Aghdasi, PROMETHEE: A comprehensive literature review on methodologies and applications, European Journal of Operational Research, 200, 198-215, (2010).

[8] B. Mareschal, J.P. Brans, P. Vincke, et al., PROMETHEE: A new family of outranking methods in multicriteria analysis. Technical Report. ULB-Universite Libre de Bruxelles (1984).

[9] G. Büyüközkan, F. Göçer, Application of a new combined intuitionistic fuzzy mcdm approach based on axiomatic design methodology for the supplier selection problem. Applied Soft Computing 52, 1222-1238, (2017).

[10] M. Çitil, F. Tuğrul, B. Yılmaz, An Application of Multi Criteria Decision Making: Ranking of School Success, Celal Bayar University Journal of Science, 15(1), 45-50, (2019).

[11] N. Halouani, H. Chabchoub, J.M. Martel, Promethee-md-2t method for Project selection. European Journal of Operational Research 195, 841-849, (2009).

[12] R. Krishankumar, K. Ravichandran, A.B. Saeid, A new extension to promethee under intuitionistic fuzzy environment for solving supplier selection problem with linguistic preferences. Applied Soft Computing 60, 564-576, (2017).

[13] H. Liao, Z.S. Xu, Multi-criteria decision making with intuitionistic fuzzy PROMETHEE, Journal of Intelligent Fuzzy Systems, 27, 1703-1717, (2014a).

[14] H. Liao, Z. Xu, Priorities of intuitionistic fuzzy preference relation based on multiplicative consistency. IEEE Transactions on Fuzzy Systems 22, 1669-1681, (2014b).

[15] H. Liao, Z. Xu, Some algorithms for group decision making with intuitionistic fuzzy preference information. International Journal of Uncertainty, Fuzziness and Knowledge- Based Systems 22, 505-529, (2014c).

[16] M. Majumder, Multi Criteria Decision Making. Chapter 2, Springer, (2015).

[17] E. Szmidt, J. Kacprzyk, Amount of information and its reliability in the ranking of Atanassov's intuitionistic fuzzy alternatives, in: E. Rakus-Andersson, R.R. Yager, N. Ichalkaranje, L. Jain, ed. Recent advances in decision making (Studies in Computational Intelligence), Berlin, Germany: Springer, (2009).

[18] Z. Wang, Derivation of intuitionistic fuzzy weights based on intuitionistic fuzzy preference relations, Applied Mathematical Modelling, 37, 6377-6388, (2013).

[19] Z.S. Xu, R.R. Yager, Some geometric aggregation operators based on intuitionistic fuzzy sets. International journal of general systems 35, 417-433, (2006).

[20] Z.S. Xu, Intuitionistic fuzzy aggregation operators, IEEE Transactions on Fuzzy Systems, 15, 1179-1187, (2007a).

[21] Z.S. Xu, Intuitionistic preference relations and their application in group decision making, Information Sciences, 177(11), 2363-2379, (2007b). 
[22] Z.S. Xu, H. Liao, Intuitionistic fuzzy analytic hierarchy process. IEEE transactions on fuzzy systems 22, 749-761, (2013).

[23] L.A. Zadeh, Fuzzy Sets, Information and Control, 8, 338-353, (1965).

[24] K. Zhang, C. Kluck, G. Achari, A comparative approach for ranking contaminated sites based on the risk assessment paradigm using fuzzy promethee. Environmental management 44, 952, (2009).

[25] F. Tuğrul, M. Çitil, B. Karasolak, M. Dağlı, Interpretation of Physical Conditions of Schools with Fuzzy Multi Criteria Decision Making, Journal of Universal Mathematics, 3(1), 46-52, (2020).

[26] F. Tuğrul, B. Yılmaz, M. Çitil, Application of Ranking with Similarity Measure in Multi Criteria Decision Making, Konuralp Journal of Mathematics, 7(2), 438-441, (2019a).

[27] F. Tuğrul, M. Çitil, S. Balcı, On The Determination Students' Aptitude Using Intuitionistic Fuzzy Logic, Journal of Universal Mathematics, 2(1), 36-41, (2019b).

[28] F. Tuğrul, B. Yılmaz, M. Çitil, School Success Ranking in Multi Criteria Decision Making, Turkish Journal Mathematics and Computer Science, 10, 1-6, (2018).

[29] F. Tuğrul, M. Çitil, M. Gezercan, Application of Intuitionistic Fuzzy Sets in High School Determination Via Normalized Euclidean Distance Metod, Notes on Intuitionistic Fuzzy Sets, 23(1), 42-47, (2017).

Department of Mathematics, Kahramanmaraş Sütcü İmam University, Kahramanmaraş, TURKEY

Email address: feridetugrul@gmail.com,citil@ksu.edu.tr 